http://jmscr.igmpublication.org/home/ ISSN (e)-2347-176x ISSN (p) 2455-0450

crossref DOI: https://dx.doi.org/10.18535/jmscr/v8i2.29

Journal Of Medical Science And Clinical Research

\title{
Granulomatosis with Polyangitis Masquerading As Rheumatoid Vasculitis
}

\author{
Authors \\ Dr Ponambalaganapathi Nadar ${ }^{1}$, Dr Durgakrishnan ${ }^{2}$, Dr K. Mayilananthi ${ }^{3}$ \\ ${ }^{1}$ Postgraduate, Department of General Medicine, Chettinad Hospital \& Research Institute \\ ${ }^{2,3}$ Professor, Department of General Medicine, Chettinad Hospital \& Research Institute
}

\begin{abstract}
Granulomatosis with polyangitisis characterized by upper and lower respiratory tract with kidney involvement. We report a case of 32 year old male patient with clinical features resembling rheumatoid arthritis with vasculitis and oral lesion turns out to be rare presentation of Granulomatosis with polyangitis with ulcerative tonsillar lesion and Anti-PR3 (c-ANCA) positive.

Keywords: Granulomatosis with polyangitis, Rheumatoid arthritis, vasculitis, Oral ulcerative lesion, Upper respiratory tract, Lower respiratory tract, Arthritis.
\end{abstract}

\section{Case Report}

A 32 year old male patient with complain of knee and ankle joint pain for 3-4 week which was initially present while walking upstairs then progress to be while walking on the floor, associated with swelling of the joint. Patient complain of fever since 7 -10 days, which was low grade, intermittent not associated with chills and rigors, got relieved with medication associated with redness of eyes. During the course in the hospital itself, patient started getting severe pain and swelling in the small joint of hands and feet, symmetrically and along with the involvement of elbow joint, and associated with morning stiffness of more than one hour meeting EULAR CRITERIA for Rheumatoid arthritis

Patientis conscious, Oriented to time palace and person, Pulse: 70/min, BP: 110/70 mm Hg, Conjunctiva congested, Sclera red, No Icterus/cyanosis/clubbing, No lymphadenopathy. System examination was normal with no focal neurological deficit. Local examination revealed swelling warmth, tenderness of small joints of hands, feet with predominant involvement of proximal interphalangeal joint (PIP) and metacarpophalangeal (MTP) and metatarsophalangeal joint (MTP). Large joint like Knee and ankle joint was also involved associated with tenderness and restricted in normal joint movement.

Laboratory value of normal routine test as below:

Chest X-ray was normal. RA factor and CRP was reactive with titre around $96 \mathrm{IU} / \mathrm{L}$ and121.63. RA factor was elevated more than 3 times the upper level of normal. Patient was started on injectable steroid, oral Hydroxychloroquine (HCQ) and oral Methotrexate, during 2 days of steroid, patient's joint pain and redness of eye decreased substantially, while he improved symptomatically. Onday 3 of steroid, patient developed 2 episodes of hematemesis with frank blood.

Upper gastrointestinal endoscopy was done under sedation which is suggestive of growth in oropharynx and adjacent left tonsillar area with 
oesophagus gastro duodenal reflex and erosiveduodenitis. Patient was started on pantoprazole infusion and patient had no further episodes of haematemesis.

Video laryngoscopy (VL scopy) was done in view of oropharynx and tonsillar lesion. VL scopy suggestive of ulceration over anterior pillar, tonsillar surface, left tonsillar surface looks irregular, slough covered with pooling of saliva, while other neighbouring structure like larynx, pharynx and pyriform fossae appears normal. Below is the depicted picture of VL scopy with left tonsillar surface lesion.

\begin{tabular}{|l|c|c|c|}
\hline HB & $\mathbf{1 1 . 8}$ & Albumin & + \\
\hline Platelet & 4.3 lac & Pus cells & $1-2$ cells \\
\hline RBS & 103 & Epi cell & 3 to 4 \\
\hline Urea/creatinine & $11 / 0.79$ & Ketone & Negative \\
\hline Sodium/potassium & $138 / 5.4$ & RBC & negative \\
\hline Serology for HIV/HBV/HCV & Negative & Urine Culture & negative \\
\hline ESR & $120 \mathrm{~mm}$ & Spot PCR & $30 \mathrm{mg} / \mathrm{mmol}$ \\
\hline LFT & Normal except A/G reversal & & \\
\hline & $\begin{array}{l}\text { Albumin:3.5 } \\
\text { Total protein:9.3 }\end{array}$ & \\
\hline
\end{tabular}
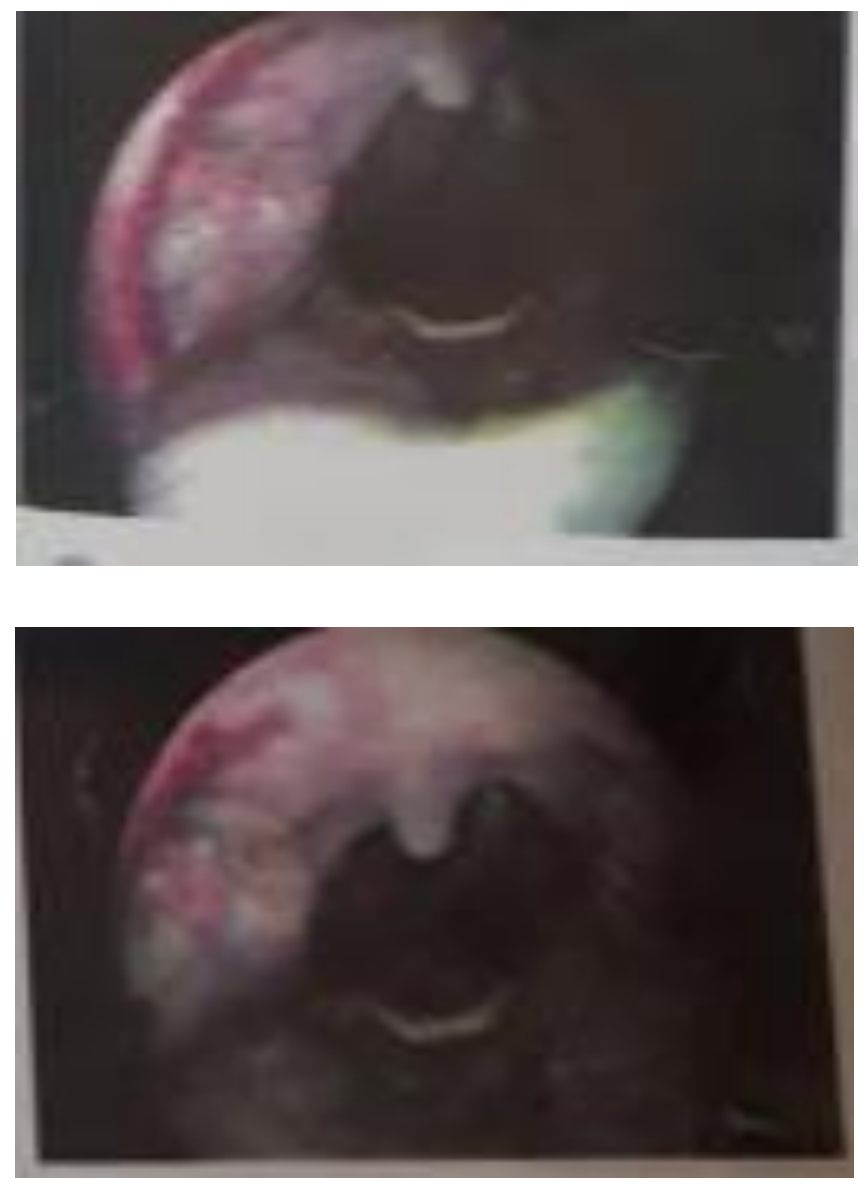

In meantime patient was found to be negative for ANA, Anti-CCP and C3 and C4 were normal. CANCA (anti PR-3) was positive, and patient was started on azathioprine and steroid was continued, patient improved symptomatically. HRCT was normal.

\section{Discussion}

Granulomatosis with polyangiitis which was initially called as rhinogenic granulomatis present with nasal or oral lesion as initial presentation with due course of time typical picture of Granulomatosis with polyangiitis develops. Here we report case, were patient was initially presented clinically as rheumatoid arthritis with vasculitis feature, subsequently developing oral lesion. Rarely which could be the initial picture of Granulomatosis with polyangiitis. Granulomatosis with polyangitis typically present with upper and lower respiratory involvement associated renal involvement. Though upper respiratory and lower 
respiratory tract is involved, it is the predominant nasal lesion which present as initial lesion while the oral cavity involvement of tonsil is less than 3 $\%$. In our case scenario, patient present with poly arthritis associated with early morning stiffness initially, typical of rheumatoid arthritis. Sclera involvement which can thought to be an extra articular manifestation of rheumatoid arthritis, but in due course of time patient presented with oral ulcerative lesion involving tonsillar region which could be starting point for Granulomatosis with polyangiitis.

Patient presenting with oral ulcerative lesion of tonsil, sclera/episcleral involvement, arthritis and C-ANCA positive, with improvement from steroid and azathioprine suggestive of Granulomatosis with polyangiitis.

\section{Conclusion}

Granulomatosis with polyangiitis can be rarely presenting with initial picture of rheumatoid arthritis with subsequently evolving into Granulomatosis with polyangiitis as oral ulcerative tonsil lesion as initial sign. Hence early examination oral and nasal cavity would have revealed some granulomatous tissue, which would have help to early diagnosis of Granulomatosis with polyangiitis.

\section{References}

1. Genuis, K., Pewarchuk, J. Granulomatosis with polyangiitis (Wegener's) as a necrotizing gingivitis mimic: a case report. J Med Case Reports 8, 297 (2014) doi:10.1186/1752-1947-8-297

2. Jereen Varghese, Krishnapriya R, R. Srinivasan. Granulomatosis with Polyangiitis - A case report. IAIM, 2016; 3(6): 207-210.

3. Friedmann I. Wegener's granulomatosis presenting as rheumatoid arthritis. Proc R Soc Med. 1976;69(10):785.

4. Frankel SK, Cosgrove GP, Fischer A, Meehan RT, Brown KK. Update in the diagnosis and management of pulmonary vasculitis. Chest, 2006; 129(2): 452-465.

5. Jennette J.C. Nomenclature and classification of vasculitis: lessons learned from granulomatosis with polyangiitis (Wegener's granulomatosis) Clinical and Experimental Immunology. British Society for Immunology, Clinical and Experimental Immunology, 2011; 164 (Suppl. 1): 7-10. 